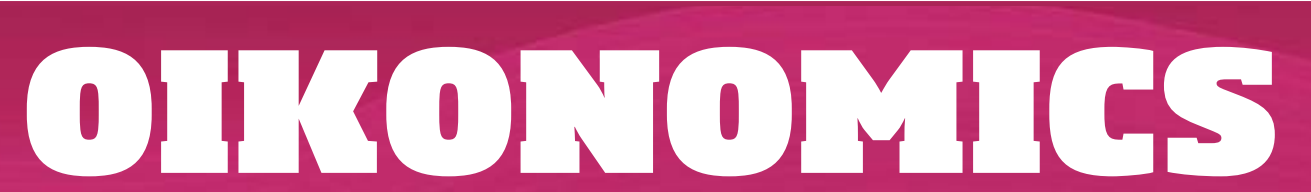

Revista de economía, empresa y sociedad

INCORPORACIÓN DE VALORES

\title{
Fl objetivo de dirigir empresas es crear justicia
}

\section{Natàlia Gugueró-Escofet}

Profesora de los Estudios de Economia y Empresa (UOC)

\section{Josep Maria Rosanas}

Profesor emérito de Contabilidad y Control (IESE)

RESUMEN La justicia siempre se ha considerado un concepto alejado de la dirección de empresas y solo se ha considerado legítimo como objetivo social. La teoría económica no ha elaborado nunca nada que sea específico para organizaciones. En concreto, uno de los padres de la economía, Adam Smith, quería ver lo qué era bueno para el mundo, que se puede equiparar a la eudaimonia que proponía Aristóteles. Pero en una visión simplista de la economía, la «mano invisible» implica que las empresas tienen que maximizar los beneficios y que al hacerlo ya contribuyen a esta eudaimonia social. Este es su rol. Pero si repensamos este objetivo propuesto por Smith, veremos que esta eudaimonia solo se puede conseguir si, cuando se considera a las empresas y su toma de decisiones, se incorporan valores y virtudes, y que esto significa ver qué objetivos tiene que proponerse la toma de decisiones y considerar los beneficios como resultados que solo llegarán si se toman las decisiones correctas, es decir, con la presencia de dichos valores. Aquí queremos exponer el hecho de que generar justicia tiene que ser uno de los objetivos básicos a la hora de dirigir una empresa, pues si no se incorpora este elemento en la toma de decisiones, la posibilidad de incorporar injusticias en las decisones que se adopten puede acabar con la misma organización. En las empresas, pues, la toma de decisiones tiene que generar justicia, y por lo tanto no se puede dirigir de cualquier manera. La justicia se tiene que aplicar con sabiduría práctica 
aristotélica, es decir, caso a caso y situación a situación. De esta manera, se podrán llegar a generar unos beneficios, que nunca se sabrá si son máximos, pero sí satisfactorios, y por lo tanto conseguir el último objetivo, que es la eudaimonia. Así coincidiría lo que decía Aristóteles con lo que proponía Adam Smith.

PALABRAS CLAVE dirección; liderazgo; gobernanza; justicia; sabiduría práctica; shareholder-value

\section{The objective of managing organizations is to generate justice}

ABSTRACT Justice has always been considered a concept that is very separate from business management and has only been considered legitimate as a social objective. Economic theory has never produced anything that is specific to organizations. In particular, one of the fathers of economics, Adam Smith, wanted to show what was good for the world, which can be paralleled with the concept of eudaimonia (happiness) proposed by Aristotle. In a simplistic view of economics, the 'invisible hand' implies that companies must maximize profits and that doing so would already contribute to this social eudaimonia. That is their role. Rethinking this objective proposed by Smith, we can see that eudaimonia can only be achieved if, when companies are analysed, their decision-making incorporates values and virtues. This requires them to decide the goals they have to set and consider the benefits as results that will only be realised by making the right decisions or, in other words, with the inclusion of these values. In this essay, our aim is to show that generating justice must be one of the basic objectives when running a company because, if this objective is not incorporated within the decision-making process, the decisions made incorporating injustices can result in the same organizational structure. For companies, therefore, decision-making must generate justice and, as such, it cannot be directed in any way. This justice must be applied with Aristotelian logic or, in other words, on a case to case basis that judges each situation on its individual merits. This is how you can generate profits, which you will never be able to ascertain whether or not they have been maximized, but which are satisfactory, thereby achieving the ultimate goal of eudaimonia. This would align what Aristotle said with what Adam Smith proposed.

KEYWORDS management; leadership; governance; justice; practical wisdom shareholder-value 


\section{Introducción}

La justicia siempre ha sido contemplada como un concepto alejado de la dirección de empresas y solo se ha considerado legítima como objetivo social. De hecho, si nos fijamos en la teoría económica, muy influyente a la hora de alimentar las teorías organizativas, y en concreto en lo que proponía Adam Smith, estaba más interesado en estudiar qué era bueno para el mundo, para llegar a la idea de cómo conseguir aquello que se podría equiparar a la eudaimonia que proponía Aristóteles. Pero la manera de trasladarlo a las empresas se ha simplificado mucho y, en realidad, la teoría económica les ha asignado el rol de generadoras de beneficios, cuantos más mejor, es decir, maximizarlos.

Pero si repensamos el objetivo que propuso Adam Smith, podemos ver que la eudaimonia que proponía solo se puede conseguir si incorporamos valores, si nos fijamos en los objetivos de cada decisión, y no solo en un potencial resultado macro del conjunto de decisiones. La eudaimonia solo se puede conseguir si somos conscientes, en el proceso de toma de decisiones, de cuáles deben ser los objetivos de las decisiones y qué conocimiento es necesario para incorporar tales objetivos. Esto hace necesario entender que deben ser los valores y las virtudes los que alimenten las decisiones y los criterios para decidir, es decir, valorar en cada situación y en cada caso particular qué conocimiento es necesario para tomar una decisión, con qué objetivo y entender el beneficio como un resultado que llegará, y de manera sostenida, si se cumplen unos determinados objetivos. Aquí mostraremos que la economía no puede ser aplicada de forma aislada a las empresas, puesto que no les proporciona un objetivo claro. Examinaremos cómo se ha aplicado y cuáles han sido sus limitaciones. A continuación, mostraremos que el foco debe estar puesto en el proceso de toma de decisiones, porque el objetivo de las empresas tiene que ser generar justicia, la única manera de conseguir que la empresa tenga sentido para todos los grupos de interés y sea sostenible a la vez que obtiene resultados económicos. También mostraremos que para tomar decisiones justas es necesario incorporar la sabiduría práctica propuesta por Aristóteles. Por lo tanto, cuando el proceso de toma de decisiones incorpora la justicia como objetivo y la sabiduría práctica como conocimiento, es cuando los beneficios que se generan son sostenibles, y la empresa puede generar el objetivo social último deseado de la eudaimonia. 


\section{Las empresas tienen un objetivo y no es el que la gente cree. La economía aplicada a la empresa y sus limitaciones}

La justicia es un concepto que en general se ha olvidado para quien diseña sociedades o sistemas sociales. Se ha considerado que las sociedades tienen que ser justas, y en esta dirección se han ido desgranando diferentes conceptos sobre qué quiere decir que sean justas. Algunos conceptos de justicia social se han considerado más adecuados que otros y, en general, cada cual ha ido exponiendo las bondades de las diferentes opciones propuestas. La más influyente en dirección de empresas ha sido la de Rawls, contrapuesta a la de Senn (Rawls, 1971, 2003; Sen, 1992). No es el objetivo de este artículo decidir cuál es el mejor concepto, pero todos estaríamos de acuerdo en que cualquier sociedad tiene que garantizar unos mínimos de justicia. Una sociedad tiene que generar justicia para sus ciudadanos, pero no como última finalidad, sino porque cada cual se pueda desarrollar y pueda llegar a un estado personal de eudaimonia.

Este concepto, la eudaimonia (Aristóteles, 2009, Book I), que se suele traducir como felicidad, pero que es más amplio, lo proponen desde Aristóteles, de manera explícita, hasta Adam Smith, de manera menos explícita. Para llegar a conseguirlo, es necesario que se tomen en consideración los valores. No es posible conseguirlo sin que entren en juego los valores, que en términos aristotélicos toman el nombre de virtudes, y fundamentalmente sin que se garanticen unos ciertos niveles de justicia (Aristóteles, 2009, Book V).

Pero entonces, si para adquirir eudaimonia es necesario que se garanticen unos niveles de justicia social, es importante examinar dónde quedan las empresas en la generación de esta justicia. Y podemos observar que, de manera general, han quedado al margen, sobre todo en las visiones de la teoría económica clásica aplicadas a la empresa. Las empresas y su manera de funcionar han sido simplificadas por las teorías más influyentes, especialmente las económicas, que son las que queremos desgranar de manera especial aquí. El problema de cómo se aplica la teoría económica es que no tiene objetivo, y menos para las empresas, puesto que solo les atribuye la función de generar los máximos beneficios posibles sin concretar de qué manera y para qué.

Desde este punto de vista, lo que deberían hacer las empresas es generar riqueza, y después ya serán los «mercados» y quizás los instrumentos de gobierno de la sociedad los que se encargarán de ver cómo esta riqueza puede acabar trasladándose a los ciudadanos. Por lo tanto, la función de las empresas se reduciría a generar riqueza, cuanta más mejor, primero para los accionistas y después para la sociedad. El hecho de decidir en qué proporción tienen que contribuir a la 
riqueza colectiva ya será un tema de modelo de sociedad y de concepto de justicia predominante.

Pero hay dos cuestiones que hacen que esta simplificación no funcione. La primera es la confusión entre objetivos y resultados. Claramente no son lo mismo, y la economía los confunde en muchas ocasiones, lo que nos ha llevado a situaciones realmente caóticas, como la crisis que se debe a aquellos sistemas de incentivos guiados para maximizar los resultados (Cugueró-Escofet y Rosanas, 2016). Los objetivos se plantean antes y los resultados vienen después como consecuencia de conseguir determinados objetivos y de una determinada manera particular.

Y la segunda cuestión es que la teoría económica señala que las empresas tienen que generar resultados, pero no dice nada sobre cuáles tienen que ser sus objetivos; por lo tanto, en este sentido, no ayuda a desarrollar una teoría realista de dirección de empresas que permita guiar la acción directiva a medio y largo plazo. Las empresas reales sí que tienen que tener objetivos. Ahora bien, para saber cuáles son y cómo desarrollarlos no nos podemos basar en lo que dice la economía. Y es precisamente esto lo que haremos a continuación, examinar por qué la economía, tal y como se aplica mayoritariamente, no es útil para ayudar a determinar cuáles son los objetivos de las empresas.

\subsection{Limitaciones de la teoría económica}

Se puede considerar que la influencia de la economía en la dirección de empresas empezó a verse en áreas como finanzas y contabilidad, pero ha continuado con la estrategia e incluso la dirección de personas.

Los modelos económicos tienen una manera de ver a las personas, puesto que asumen que estas tienen una racionalidad ilimitada, que en general solo se mueven por intereses personales y egoístas, y que no aprenden. Estos presupuestos no son en realidad nada realistas cuando se aplican a las empresas y sus modelos de decisión, sino que más bien son promotores de decisiones que acaban generando resultados contraproducentes a medio y largo plazo (Rosanas, 2011).

En primer lugar, asumir una racionalidad ilimitada es muy poco realista y, por lo tanto, totalmente inútil para crear modelos de decisión que puedan ser aplicables a la empresa. En general, las personas no pueden prever todas las posibilidades futuras en toda circunstancia, ni tampoco saber perfectamente cuáles son sus preferencias en todo momento o tener en la mente todas las alternativas posibles.

En segundo lugar, si se acepta que el objetivo social que deben tener las organizaciones es maximizar su valor para los accionistas, la empresa se reduce a un problema de agencia, en el que hay un principal (posiblemente los accionistas) y el resto de los elementos de interés quedarían agrupados en un único grupo 
de agentes. Los agentes son típicamente considerados egoístas, y su objetivo es maximizar su propia función de utilidad. Y aquí es cuando aparece el primer problema y limitación, puesto que considerar que los agentes son egoístas puede acabar conduciendo a que acaben siéndolo, aunque originalmente no fuera este el caso, la llamada profecía que se ha autocumplido (Ghoshal, 2005).

Finalmente, una tercera limitación es considerar que las personas no aprenden. En realidad, según Rosanas (2008), cuando dos personas interactúan pasan tres cosas. La primera son los resultados explícitos de la interacción, que pueden ser los esperados o no. En segundo lugar, se produce un aprendizaje personal de cada individuo, puesto que todos aprenden a ver si aquellos resultados esperados satisfarán mejor o no en el futuro las mismas expectativas respecto a cómo lo han hecho en el caso presente. Y finalmente, la satisfacción que se ha producido al interactuar con el otro, que hace que en el futuro tengamos más o menos deseos de repetir la interacción.

\section{La justicia como objetivo de dirigir empresas}

No obstante, la riqueza que generan las empresas no es un objetivo, es un resultado que llega cuando estas funcionan como deben hacerlo, es decir, con los objetivos correctos. Y para decidir cuáles son los objetivos correctos, debemos tener en cuenta que son aquellos que nos permitan satisfacer de manera sostenida a todos los grupos de interés, no solo a los accionistas y, por lo tanto, cambiar la «maximización de valor por los accionistas» para «satisfacer las necesidades de todos de manera equilibrada», es decir, justa. Y con este ánimo, tomando las decisiones adecuadas, una a una, de acuerdo con cada situación que se plantee, sin automatismos.

Aquí estamos interesados en mostrar que la justicia tiene que ser un objetivo también de las empresas. Generar riqueza en las empresas tendría que ser el resultado de hacer las cosas siguiendo unos criterios de justicia, porque solo así son sostenibles, y la riqueza que generan llega y lo hace no sólo a corto plazo sino también a medio y largo plazo. Y en segundo término entender que las empresas tienen que gestionar no sólo la generación de justicia a través de sistemas que en la toma de decisions la permitan, sino también las percepciones de las personas que interactúan con estas deciones.

\subsection{Justicia en la toma de decisiones}

Los principales sistemas en las organizaciones que ayudan a tomar decisiones y a diagnosticar los resultados de las empresas son los sistemas de control de ges- 
tión (Anthony y Govindarajan, 2003). En general, estos sistemas persiguen que las personas tomen las decisiones que les favorecen y, al mismo tiempo, favorezcan también a la empresa en su conjunto, es decir, generen congruencia de objetivos (goal congruence, en el término inglés más conocido) (Vancouver \& Schmitt, 1991). Esta congruencia de objetivos, junto con la fairness, serían aspectos básicos a la hora de examinar que un sistema de control sea eficaz (Vancil, 1973). Últimamente, la justicia, como variable con sus dos dimensiones, formal e informal (toma de decisiones), se ha considerado un requisito para garantizar el máximo nivel de congruencia de objetivos (Cugueró-Escofet y Rosanas, 2013), pero sobre todo para garantizar que los resultados percibidos sean justos (Cugueró-Escofet y Rosanas, 2015)

\subsection{Las percepciones de justicia}

Por lo que respecta a las políticas de recursos humanos, en algunas escuelas de pensamiento, las percepciones de justicia se han considerado básicas para evaluar el clima y para que las personas que trabajan en la empresa se sientan bien tratadas e integradas. Las teorías de justicia organizativa estudian las percepciones de justicia relacionadas con: los procesos formales (procedural justice), lo que reciben las personas (distributive justice), la manera en que se transmite la información (informational justice) y cómo se produce cada una de las interacciones (interpersonal justice), las cuatro dimensiones importantes a la hora de valorar si hay o no justicia en la manera como me tratan en una empresa (Cropanzano, Bowen y Gilliland, 2007; Greenberg, 1990). Aunque la justicia organizativa se fije en las percepciones, y estas no sean necesariamente elementos fieles para valorar si existe o no justicia, sí que es cierto que son importantes para saber si la justicia que se quiere generar realmente se percibe como tal o si, aunque creamos que los sistemas son justos, en realidad no lo están siendo (Cugueró-Escofet y Fortin, 2014). Por lo tanto, generar justicia mediante sistemas de control y comprobar que se percibe desde el punto de vista de los afectados sería la manera correcta de diagnosticar que los objetivos de crear justicia se consolidan y las decisiones se están tomando de manera correcta.

\section{Tomar decisiones justas incluye la sabiduría práctica aristotélica}

Para hacerlo hay que tomar decisiones justas por medio del conocimiento que nos proporciona la sabiduría práctica. Es importante entender que las decisiones toma- 
das afectan a las personas, implican actuar y generan consecuencias. A la hora de tomar una decisión, es importante intentar conocer todas las circunstancias que la rodean. Pero el conocimiento real total nunca es posible, por lo que se necesita el conocimiento práctico que implica poder predecir de forma individualizada las potenciales consecuencias de cada una de las decisiones que el directivo tiene que tomar. Es decir, evaluar las acciones antes de llevarlas a la práctica y hacerlo para cada persona a quien afectará esa decisión y esas acciones. Así pues, es necesario utilizar el conocimiento práctico para tomar decisiones que tengan como objetivo crear justicia para todo el mundo, en la medida en que esto sea posible.

Pero la realidad es que en dirección de empresas es muy frecuente que se busquen recetas, por lo que las propuestas que las propugnan y defienden tienen mucho éxito. Recetas «automáticas», que no requieren pensar y que un ordenador puede proporcionar. Es mucho más cómodo, e incluso se puede pensar que más eficaz. Y en algunas ocasiones puede serlo a corto plazo, en decisiones concretas, pero un desarrollo a largo plazo de una organización que tenga una ventaja competitiva sobre las otras requiere una sabiduría práctica, referida a las cosas concretas que tienen que ver con esa organización. Y precisamente porque es así, traerlo a la práctica necesitará virtudes, la primera de las cuales es la justicia.

\section{Conclusiones}

En este artículo hemos examinado y desgranado cómo se vincula la justicia social con la justicia en las empresas. En general, las dos se han desvinculado en economía. En el proceso de generación de justicia social, las empresas son consideradas como meros generadores de recursos, y son luego los mercados o los sistemas de gobierno los que se encargan de distribuirlos, y, en alguna medida, esta distribución, si sigue ciertos criterios de justicia, acabará generando la eudaimonia aristotélica. Pero la realidad es que esta explicación de la economía presenta limitaciones. En primer lugar, porque se confunden resultados con objetivos. Los resultados vienen tras plantearse unos objetivos que los generen. $Y$ en segundo lugar, porque la teoría económica no habla en ningún momento de cuáles deberían ser los objetivos de las empresas, y ya acabamos de comentar que ese objetivo último de generar resultados no guía las decisiones. Finalmente, hay otros análisis que también ponen limitaciones a las teorías económicas aplicadas a la toma de decisiones, dado que se basan en un modelo de agencia que no es realista y presenta tres limitaciones básicas. Las limitaciones serían considerar una racionalidad ilimitada que no es realista, además de considerar que los motivos de los agentes siempre son egoístas, y que estos no aprenden nunca. En estas tres limitaciones hacen que su teoría no sea aplicable a las empresas. Concluimos que una manera 
de superar estas limitaciones es considerar que la toma de decisiones tiene que tener como objetivo generar justicia. Proponemos que la justicia tiene que alimentar los sistemas formales de control de gestión, tiene que ser una virtud de los directivos y, a la vez, se tiene que procurar que las personas reciban aquello que perciben como justo. Y todo eso -incorporar la justicia como objetivo, como valor que guía las decisiones- debe complementarse con un conocimiento práctico aplicado a cada caso, que es la sabiduría práctica aristotélica.

\section{Referencias bibliográficas}

ANTHONY, R. N.; GOVINDARAJAN, V. (2003). Management Control Systems. 11. a ed. Homewood, Illinois: Richard D. Irwin.

ARISTÓTELES (2009). The Nicomachean Ethics (W. D. Ross, revisado por Leslie Brown). Oxford:. Oxford University Press.

CROPANZANO, R.; BOWEN, D. E.; GILLILAND, S. W. (2007). «The Management of Organizational Justice». Academy of Management Perspectives. Vol. 21, n. ${ }^{\circ}$ 4, págs. 34-48.

CUGUERÓ-ESCOFET, N.; ROSANAS, J. M. (2013). «The just design and use of Management Control Systems as requirements for Goal Congruence». Management Accounting Research. Vol. 24, n. ${ }^{1}$, págs. 23-40.

CUGUERÓ-ESCOFET, N.; FORTIN, M. (2014). «One Justice or Two? A Model of Reconciliation of Normative Justice Theories and Empirical Research on Organizational Justice». Journal of Business Ethics [artículo en línea]. Vol. 124, n. ${ }^{\circ}$, págs. 435-451. <http://dx.doi.org/10.1007/s10551-013-1881-1>.

CUGUERÓ-ESCOFET, N.; ROSANAS, J. M. (2015). "Social dynamics of Justice: The Ex-ante and Ex-post Justice Interplay with Formal and Informal Elements of Management Control Systems». En: Dirk, D. S.; GILLILAND, S. W.; SKARLICKI, D. P. (eds.). Social dynamics of organizational justice. Charlotte, NC: Information Age Publishing.

CUGUERÓ-ESCOFET, N.; ROSANAS, J. M. (2016). «The Ethics of Metrics: Overcoming the Dysfunctional Effects of Performance Measurements Through Justice». Journal of Business Ethics [artículo en línea]. <http://dx.doi.org/10.1007/ s10551-016-3049-2>.

GHOSHAL, S. (2005). «Bad Management Theories are Destroying Good Management Practices». Academy of Management Learning and Education. Vol. 4, n. ${ }^{\circ}$ 1, págs. 75-91.

GREENBERG, J. (1990). «Organizational justice: Yesterday, today, and tomorrow». Journal of Management. Vol. 16, n. ${ }^{\circ}$ 2, págs. 399-432. 
RAWLS, J. (1971). A theory of justice. Cambridege, MA: The Belknap press of Harvard University Press.

RAWLS, J. (2003). Justice as Fairness: a restatement. Cambridege, MA: The Belknap press of Harvard University Press.

ROSANAS, J. M. (2008). «Beyond Economic Criteria: A Humanistic Approach to Organizational Survival». Journal of Business Ethics. Vol. 78, n. ${ }^{\circ}$ 3, págs. $447-$ 462.

ROSANAS, J. M. (2011). «A Humanistic Approach to Organizations and Organizational Decision-making». En: The Future of Leadership Development (págs. 143-176). Londres: Palgrave Macmillan.

SEN, A. (1992). Inequality Reexamined. Cambridge, MA: Harvard University Press. VANCIL, R. F. (1973). «What kind of management control do you need?». Harvard Business Review.

VANCOUVER, J. B.; SCHMITT, N. W. (1991). «An exploratory examination of person-organization fit: Organizational goal congruence». Personnel Psychology. Vol. 44, n. ${ }^{\circ}$ 2, págs. 333-352. 


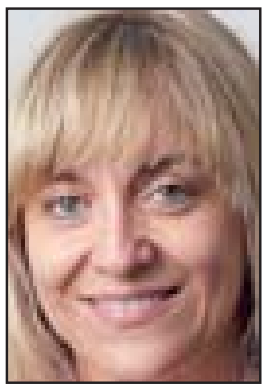

\section{Natàlia Gugueró-Fscofet ncuguero@uoc.edu \\ Profesora de los Estudios de Economía y Empresa (UOC)}

Ph.D en Management Sciences por la IESE Business School, Universidad de Navarra, Licenciada en Derecho por la UOC e Ingeniera Industrial por la UPC. Ha publicado varios artículos tanto en revistas especializadas académicas cómo en revistas de divulgación.

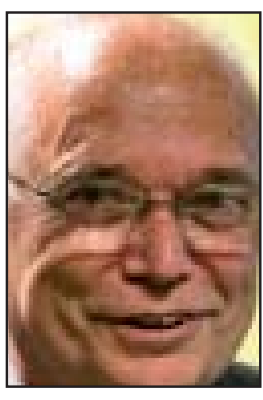

\section{Josep Maria Rosanas Martí} jrosanas@iese.edu Profesor emérito de Contabilidad y Control (IESE)

Doctor Ingeniero Industrial, MBA por el IESE y PhD en Management por la Northwestern University. Ha sido Catedrático de la Universitat Pompeu Fabra y formó parte del equipo fundador de esta Universidad. Ha publicado una veintena de libros y muchos artículos, tanto en revistas especializadas académicas cómo en revistas de divulgación y destinadas a un público profesional.

Los textos publicados en esta revista están sujetas -salvo que se indique el contrario- a una licencia de Reconocimiento 3.0 España de Creative Commons. Podéis copiarlos, distribuirlos, comunicarlos públicamente y hacer obras derivadas siempre que reconozcáis los créditos de las obras (autoría, nombre de la revista, institución editora) de la manera especificada por los autores o por la revista. La licencia completa se puede consultar en http://creativecommons.org/licenses/by/3.0/es/deed.ca.

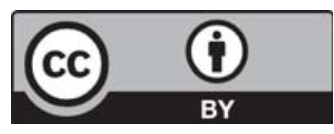

